\title{
Análise do perfil clínico- epidemiológico da hanseníase no Pará e avaliação dos indicadores de saúde
}

\author{
Analysis of the epidemiological and clinical profile of leprosy at Para and \\ assessmenting health indicator
}

\section{Análisis del perfil epidemiológico de la lepra em Pará y uma análise de los indicadores de salud}

Carla Andrea Avelar Pires ${ }^{1,2}$, Emanuele Cordeiro Chaves ${ }^{1}$, Caio Fonseca Salmen², Anthony Benny da Rocha Balieiro ${ }^{*}$, Marcela Bianca Lima Santos², Gilson Guedes de Araújo Filho1, Fernando Monteiro Mesquita ${ }^{3}$, Sabrina Sampaio Bandeira ${ }^{4}$, Marília Brasil Xavier ${ }^{1,2}$.

\section{RESUMO}

Objetivo: Analisar os indicadores de saúde e perfil clínico epidemiológico da incidência de hanseníase no Pará nos anos de 2010 a 2015. Métodos: É uma pesquisa de caráter descritivo, transversal e retrospectiva. Foi baseada por meio de levantamento do banco de dados do Sistema de Informação de Agravos de Notificação, com registros de indivíduos de casos novos de hanseníase no estado do Pará durante o período analisado. Resultados: Pode-se notar que há um predomínio de indivíduos do sexo masculino acima dos 15 anos, com classificação operacional multibacilar e forma clínica dimorfa. A taxa de detecção teve sua classificação reduzida de "hiperendêmica", até 2014, para "muito alta" em 2015. Também houve redução da classificação da prevalência de "alta" para "média". Verificou-se, porém, que a taxa de detecção em menores de 15 anos, conhecido como evento sentinela, permaneceu classificado como "hiperendêmico" durante toda a série histórica analisada. Conclusão: Conclui-se que mesmo com a redução da classificação de alguns dos indicadores de saúde, os mesmos continuam em patamares elevados para os padrões da Organização Mundial de Saúde e maiores que a média nacional, inclusive com número importante de casos em menores de 15 anos.

Palavras-Chave: Hanseníase, Indicadores Básicos de Saúde, Aplicações da Epidemiologia.

\begin{abstract}
Objective: Review of the indicators for health and clinical epidemiological surveillance of the incidence of leprosy in the state of Pará, in the years from 2010 to 2015. Methods: This is a survey of the nature, descriptive, cross-sectional, and retrospective. It was based on, by means of a survey of a database of the Information System on Disease Notification, registration of individuals with new cases of leprosy in the brazilian state of Pará, during the period under review. Results: It may be noted that there is a preponderance of males over the age of 15, with multibacillary operational classification and dimorphic clinical form. The coefficient of detection had its classification reduced from "hyperendemic" until 2014 to "very high" in 2015. There was also a reduction in the classification of the prevalence from "high" to "average". It was verified, however, that the detection coefficient in children under. Conclusion: 15 years old remained classified as "hyperendemic" throughout the historical series analyzed. It was thus realized that even with the reduction of the classification of some of the health indicators, they are still at high levels according to World Health Organization standards, including a significant number of cases in children under 15 years.
\end{abstract}

Key words: Leprosy, Health Status Indicators, Uses of Epidemiology.

\footnotetext{
1 Universidade Federal do Pará (UFPA), Belém-PA. *E-mail: anthonybenny1996@outlook.com

2 Universidade do Estado do Pará (UEPA), Belém-PA.

${ }^{3}$ Centro Universitário do Estado do Pará (CESUPA), Belém-PA.

4 Secretaria de Estado de Saúde Pública do Pará (SESPA), Belém-PA.
} 


\section{RESUMEN}

Objetivo: Analizar los indicadores de salud y el perfil clínico-epidemiológico de la incidencia de la tuberculosis en el estado de Pará, en los años 2010 y 2015. Métodos: Es una investigación de carácter descriptivo, transversal y retrospectivo. Se basa, por medio de la elevación de la base de datos del Sistema de Información de Agravios de Notificación y registro de las personas, de los casos nuevos de lepra en el estado de Pará, en brasil, durante el período que se analiza. Resultados: Hay un predominio de la población masculina por encima de los 15 años, con clasificación operacional multibacilar, forma clínica dimorfa. La tasa de detección tuvo su clasificación reducida de "hiperendémica", hasta 2014, para "muy alta" en 2015. También hubo reducción de la clasificación de la prevalencia de "alta" para "media". Se verificó que la tasa de detección en menores de 15 años, evento centinela, permaneció clasificado como "hiperendémico" durante el período analizado. Conclusión: Aunque hay reducción de la clasificación de algunos de los indicadores de salud, estos siguen en niveles elevados para los patrones de la Organización Mundial de la Salud, mayores que la media nacional, incluso con un número importante de casos en menores de 15 años.

Palabras clave: Lepra, Indicadores Básicos de Salud, Aplicaciones de la Epidemiología.

\section{INTRODUÇÃO}

A hanseníase tem alto potencial incapacitante relacionado ao seu poder imunogênico. É uma doença crônica, infectocontagiosa, causada pelo Mycobacterium leprae, um bacilo álcool-ácido resistente, em forma de bastonete (BRASIL, 2014; LASTÓRIA JC e ABREU MAMM, 2012).

É um parasita intracelular obrigatório, com tropismo aos nervos periféricos, especificamente células de Schwann. É uma doença capaz de infectar grande número de indivíduos (alta infectividade), embora poucos adoeçam (baixa patogenicidade) (BRASIL, 2014; LASTÓRIA JC e ABREU MAMM, 2012; RODUIGUES LC e LOCKWOOD DN, 2011).

A classificação de Madri define os grupos polares, Tuberculóide (T) e Virchoviano (V) ou Lepromatoso (L); o grupo transitório e inicial da doença, a forma Indeterminada (I); e o instável e intermediário, a forma Borderline(B) ou Dimorfa (D) (LASTÓRIA JC e ABREU MAMM, 2012; RIDLEY DS e JOPLING WH, 1966)

No ano de 1966, surgiu uma nova classificação, na qual os pacientes são classificados em dois grupos polares no espectro imunológico, Tuberculoide e Lepromatoso; e três grupos intermediários: BorderlineTuberculoide, Borderline-Borderline e Borderline-Lepromatoso (RIDLEY DS e JOPLING WH, 1966).

A Organização Mundial de Saúde (OMS) em 1988, estabeleceu uma categorização simplificada da Hanseníase, chamada de Classificação Operacional. Esta permite agrupar os pacientes em Paucibacilares (PB), casos com até cinco lesões cutâneas e/ou um tronco nervoso acometido, e Multibacilares (MB), casos com mais de cinco lesões cutâneas e/ou mais de um tronco nervoso acometido (BRASIL, 2014; LASTÓRIA JC e ABREU MAMM, 2012; WHO, 2006).

Sua transmissão ocorre principalmente pelo trato respiratório, possuindo um período de incubação que dura em média de dois a sete anos. O longo período de incubação dificulta as ações de vigilância epidemiológica e seu diagnóstico precoce (BRASIL, 2014; LASTÓRIA JC e ABREU MAMM, 2012; WHO, 2006; BRASIL, 2007; BRASIL, 2008).

O diagnóstico é essencialmente clínico, realizado por meio da análise da história e condições de vida do paciente, além do exame dermatoneurológico para identificar lesões ou áreas de pele com alteração de sensibilidade e/ou comprometimento de nervos periféricos (sensitivo, motor e/ou autonômico) (BRASIL, 2007; ALVES ED, et al, 2014). No estado do Pará, o valor médio da taxa de detecção da hanseníase no período de 2006 a 2008 foi de 65,20/100.0000 habitantes, ressaltando-se que no ano de 2008 atingiu uma taxa de detecção de 63,77/100.000 habitantes (BRASIL, 2009).

Em 2009, a taxa de detecção foi de 5,97 casos por 10.000 habitantes, o que caracteriza uma condição de hiperendemicidade. Esta endemia está distribuída de forma heterogênea, com maior distribuição nas regiões Sul e Sudeste do estado (PALÁCIOS VRCM, et al, 2010; BRASIL, 2009). 
O Sistema de Notificação de Agravos Notificáveis (SINAN) é o sistema oficial brasileiro para toda e qualquer informação de hanseníase, esta é uma doença de notificação compulsória em todo o território nacional e investigação obrigatória (BRASIL, 2015).

O Ministério da Saúde acompanha o desempenho e o impacto das ações através da análise dos seguintes indicadores: taxa de detecção, taxa de prevalência, bem como proporção de casos, sendo o presente justamente com o objetivo de analisar os indicadores de saúde e perfil clínico epidemiológico como forma de contribuição. Somado a isso, o Ministério prioriza buscar conhecer o grau de incapacidades físicas entre os casos novos detectados e avaliados. (BRASIL, 2015)

A realização desta pesquisa proporciona, tanto aos pesquisadores quanto à comunidade científica, 0 conhecimento mais atual do perfil clínico-epidemiológico e constatação real dos indicadores epidemiológicos dos pacientes no período estudado. A partir desses dados, tornam-se mais precisas as elaborações de planos de controle da doença. Trabalhos analíticos também poderão ser futuramente, realizados com o apoio dos resultados que venham a ser adquiridos neste trabalho.

\section{MÉTODOS}

Foi uma pesquisa de caráter descritivo, transversal e retrospectiva. Foi baseada por meio de levantamento do banco de dados do Sistema de Informação de Agravos de Notificação, com registros de indivíduos de casos novos de hanseníase no estado do Pará durante o período analisado de 2010 a 2015, a fim de analisar o perfil clínico-epidemiológico, mas também a avaliação de Indicadores de Saúde.

A situação de a pesquisa ter um caráter descritivo está associada com a intenção de explorar, avaliar e detalhar os aspectos de uma situação envolvida à Hanseníase (POLIT DF, 2006).

A coleta de dados ocorreu através de um banco de dados solicitado à Direção de Vigilância à Saúde da Secretaria de Estado de Saúde Pública do Pará (SESPA). A coleta foi realizada no período entre outubro e novembro de 2017.

Este estudo faz parte de uma pesquisa maior intitulada: avaliação dos registros de preenchimento das fichas de notificação de hanseníase no Pará, aprovado no comitê de ética e pesquisa do Núcleo de Medicina tropical da Universidade Federal do Pará, sob o parecer de número 2.257.543 e todos os aspectos éticos dessa pesquisa estão de acordo com as normas estabelecidas para o desenvolvimento de pesquisa, os dados foram coletados respeitando as normas de pesquisas que envolvem seres humanos (Resolução 466/12) do Conselho Nacional de Saúde.

A amostra é composta por 21.365 pacientes confirmados como casos novos de Hanseníase no estado do Pará entre os anos de 2010 e 2015, que atenderam aos critérios de inclusão, a saber: foram inclusos no estudo os pacientes que representaram casos novos de hanseníase residentes e diagnosticados no estado do Pará entre os anos de 2010 a 2015, independentemente da forma da doença apresentada por eles e exclusão os indivíduos transferidos de outros serviços, aqueles com recidiva, ou que foram admitidos por outras formas.

As variáveis analisadas foram: todos os casos novos confirmados de Hanseníase dos residentes no estado do Pará e notificados na base de dados do Sistema de Notificação de Agravos Notificáveis estadual (SINAN) entre 2010 e 2015, foram organizados em planilhas eletrônicas para que se realizasse a determinação do perfil epidemiológico sendo levando em conta para isso a taxa de detecção geral, proporção de casos associados a incapacidades físicas, municípios com maiores números de casos, distribuição dos casos novos de Hanseníase de acordo com a faixa e formas clínicas, mas também com os tipos de saída e/ou evolução dos pacientes diagnosticados com Hanseníase.

Além disso, a identificação de problemas que necessitam ser investigados pela vigilância epidemiológica para melhor conhecimento da doença. Todos os indicadores foram avaliados segundo as orientações disponíveis nas "Diretrizes para vigilância, atenção e eliminação da hanseníase como problema de saúde pública: manual técnico-operacional". 


\section{RESULTADOS}

Os resultados dessa pesquisa estão de acordo com a OMS, em que apenas 14 países reportaram mais de mil novos casos registrados em seus territórios em 2015. O Brasil apresentou 13\% de incidência em todos os novos casos Registrados mundialmente. O Pará, por sua vez, representa $11,53 \%$ de todos os casos do país (WHO, 2016).

Ainda segundo a OMS, no Brasil a detecção da hanseníase vinha apresentando poucas variações na última década, porém em 2015 apresentou declínio considerável (WHO, 2016). Concordando com estes dados, as taxas de detecção encontrados neste estudo confirmam que no Pará a classificação da detecção foi "hiperendêmica" até 2014 e "muito alta" no ano seguinte (Tabela 1).

Assim como a taxa de detecção, a prevalência também vinha apresentando poucas variações até o último ano do período estudado, quando houve redução da classificação deste indicador de "alta" para "média" prevalência. Todos os resultados estão apresentados nas (Tabelas de 1 a 4) e (Figuras 1 e 2), conforme as pesquisas realizadas no SINAN.

Tabela 1 - Taxa de detecção geral de casos novos e na população de 0 a 14 anos, por 100.000 habitantes, e Taxa de prevalência por 10.000 habitantes no estado do Pará.

\begin{tabular}{lcl}
\hline ANO & TAXA DE DETECÇÃO GERAL * & CLASSIFICAÇÃO* $^{*}$ \\
\hline 2010 & 49,59 & Hiperendêmico \\
2011 & 50,72 & Hiperendêmico \\
2012 & 49,88 & Hiperendêmico \\
2013 & 41,94 & Hiperendêmico \\
2014 & 42,35 & Hiperendêmico \\
2015 & 37,23 & Muito Alto \\
\hline ANO & TAXA DE DETECÇÃO 0 a 14 ANOS ${ }^{* *}$ & CLASSIFICAÇÃO* \\
\hline 2010 & 17,45 & Hiperendêmico \\
2011 & 17,32 & Hiperendêmico \\
2012 & 15,77 & Hiperendêmico \\
2013 & 18,74 & Hiperendêmico \\
2014 & 17,62 & Hiperendêmico \\
2015 & 13,64 & Hiperendêmico \\
\hline ANO & CLASSIFICAÇÃO* \\
\hline 2010 & TAXA DE PREVALÊNCIA*** & Alto \\
2011 & 5,989 & Alto \\
2012 & 6,077 & Alto \\
2013 & 6,030 & Alto \\
2014 & 5,120 & Alto \\
2015 & 5,080 & Médio \\
\hline
\end{tabular}

Fonte: Secretaria de Estado de Saúde Pública do Pará (SESPA).

Legenda: *Parâmetro, segundo o Ministério da Saúde (2016): hiperendêmico ( $\geq 40,0 / 100$ mil hab.), muito alto (20,00 a 39,99/100 mil hab.), alto (10,00 a 19,99/100 mil hab.), médio (2,00 a 9,99/100 mil hab.) e baixo $(<2,00 / 100$ mil hab.).

** Parâmetro, segundo o Ministério da Saúde (2016): hiperendêmico ( $\geq 10,0 / 100$ mil hab.), muito alto (5,00 a $9,99 / 100$ mil hab.), alto (2,50 a 4,99/100 mil hab.), médio $(0,50$ a $2,49 / 100$ mil hab.) e baixo $(<0,50 / 100$ mil hab.).

*** Parâmetro, segundo o Ministério da Saúde (2016): hiperendêmico ( $\geq 20,0 / 10$ mil hab.), muito alto (10,0 a 19,9/10 mil hab.), alto (5,0 a 9,9/10 mil hab.), médio ( 1,0 a 4,9/10 mil hab.) e baixo ( $<1,0 / 10$ mil hab.). 
Tabela 2 - Proporção de casos novos de hanseníase com grau 2 de incapacidade física no momento do diagnóstico e proporção de contatos intradomiciliares examinados de pacientes que foram diagnosticados no período de 2010 a 2015 no estado do Pará.

\begin{tabular}{cll}
\hline ANO & COEFICIENTE* $^{*}$ & CLASSIFICAÇÃO* $^{*}$ \\
\hline 2010 & 4,83 & Baixo \\
2011 & 5,55 & Médio \\
2012 & 5,72 & Médio \\
2013 & 5,47 & Médio \\
2014 & 6,20 & Médio \\
2015 & 5,13 & Médio \\
\hline CONTATOS INTRADOMICILIARES EXAMINADOS & COEFICIENTE** & CLASSIFICAÇÃO* \\
\hline 2012 & 70,21 & Precário \\
2013 & 74,37 & Precário \\
2014 & 75,84 & Regular \\
2015 & 75,19 & Regular
\end{tabular}

Fonte: Secretaria de Estado de Saúde Pública do Pará (SESPA).

Legenda: *Parâmetro, segundo o Ministério da Saúde (2016): alto ( $\geq 10 \%)$, médio ( 5 a $9,99 \%)$ e baixo ( $<5 \%)$.

* *Para este indicador são excluídos os pacientes transferidos para outros estados e países e avalia-se nos anos das coortes, considerando todos os casos paucibacilares diagnosticados um ano antes da avaliação e todos os casos multibacilares diagnosticados dois anos antes da avaliação, por isso o cálculo dos anos de 2010 e 2011 tornou-se inviável.

${ }^{* * *}$ Parâmetro, segundo o Ministério da Saúde (2016): bom ( $\left.\geq 90 \%\right)$, regular $(\geq 75,0$ a $89,9 \%)$ e precário $(<75,0 \%)$.

Tabela 3 - Municípios com maiores números de casos novos no estado do Pará entre 2010 e 2015 e Taxa de detecção geral de casos novos nos municípios de maior detecção, por 10.000 habitantes, no estado do Pará entre 2010 e 2015.

\begin{tabular}{|c|c|c|c|c|c|c|c|}
\hline $\begin{array}{l}\text { MUNICÍPIOS/ } \\
\text { NÚMERO DE CASOS }\end{array}$ & 2010 & 2011 & 2012 & 2013 & 2014 & 2015 & TOTAL \\
\hline Marituba & 356 & 335 & 377 & 278 & 310 & 330 & 1986 \\
\hline Belém & 355 & 361 & 252 & 275 & 269 & 246 & 1758 \\
\hline Marabá & 199 & 203 & 204 & 216 & 192 & 192 & 1206 \\
\hline Parauapebas & 168 & 137 & 175 & 139 & 161 & 144 & 924 \\
\hline Altamira & 92 & 174 & 141 & 87 & 117 & 76 & 687 \\
\hline $\begin{array}{l}\text { MUNICÍPIOS/ } \\
\text { TAXA DE DETECÇÃO } \\
\text { GERAL }\end{array}$ & 2010 & & 2011 & 2012 & 2013 & 2014 & 2015 \\
\hline $\begin{array}{l}\text { Floresta do } \\
\text { Araguaia }\end{array}$ & 10,65 & & 6,65 & 16,94 & 10,67 & 13,68 & 19,72 \\
\hline Jacundá & 14,59 & & 24,52 & 18,68 & 7,9 & 11,41 & 12,32 \\
\hline Marituba & 32,88 & & 30,22 & 33,25 & 23,63 & 25,76 & 26,84 \\
\hline Rurópolis & 6,98 & & 13,08 & 27,58 & 17,81 & 10,96 & 8,33 \\
\hline Xinguara & 15,77 & & 15,61 & 18,12 & 9,5 & 13,42 & 10,50 \\
\hline
\end{tabular}

Fonte: Secretaria de Estado de Saúde Pública do Pará (SESPA). 
Tabela 4 - Distribuição dos casos novos de Hanseníase de acordo com a faixa etária entre 2010 e 2015.

\begin{tabular}{lllllllll}
\hline Faixa Etária & $\mathbf{2 0 1 0}$ & $\mathbf{2 0 1 1}$ & $\mathbf{2 0 1 2}$ & $\mathbf{2 0 1 3}$ & $\mathbf{2 0 1 4}$ & $\mathbf{2 0 1 5}$ & TOTAL \\
\hline $0-15$ & 523 & 535 & 515 & 582 & 539 & 446 & 3140 \\
$\begin{array}{l}\text { anos } \\
\begin{array}{l}16-30 \\
\text { anos }\end{array}\end{array}$ & 1313 & 1244 & 1219 & 938 & 951 & 849 & 6514 \\
$\begin{array}{l}31-45 \\
\text { anos }\end{array}$ & 1213 & 1339 & 1250 & 1082 & 1155 & 1105 & 7144 \\
$\begin{array}{l}46-60 \\
\text { anos }\end{array}$ & 968 & 972 & 1060 & 899 & 907 & 834 & 5640 \\
$>60$ anos & 537 & 583 & 655 & 581 & 550 & 620 & 3526 \\
\hline
\end{tabular}

Fonte: Secretaria de Estado de Saúde Pública do Pará (SESPA).

Figura 1 - Distribuição de acordo com as formas clínicas dos casos novos de Hanseníase no estado do Pará entre 2010 e 2015.

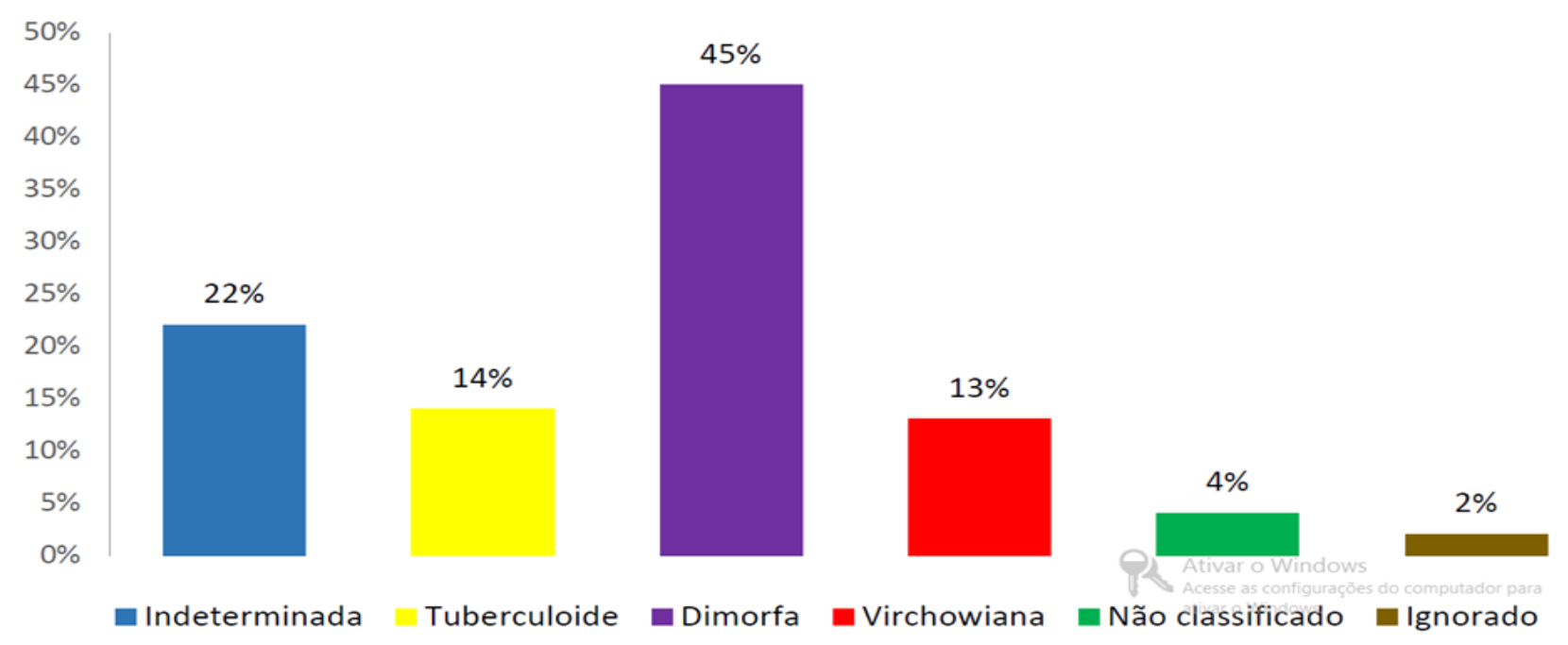

Fonte: Protocolo de pesquisa.

Figura 2 - Distribuição de acordo com os tipos de saída dos pacientes diagnosticados com Hanseníase no estado do Pará entre 2010 e 2015.

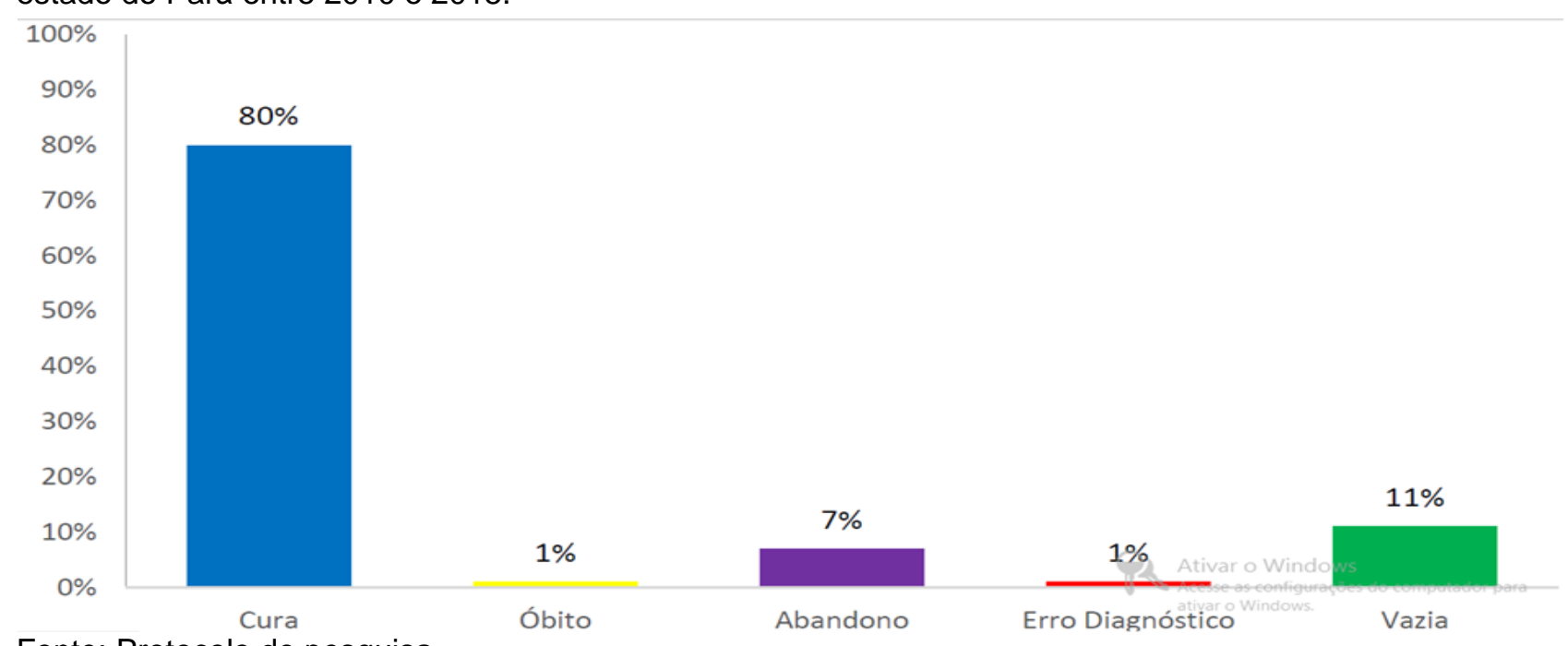

Fonte: Protocolo de pesquisa. 


\section{DISCUSSÃO}

Esta pesquisa demonstrou que no estado em questão a incidência permanece alarmante, classificada como "muito alta"; há uma alta detecção em menores de 15 anos que revela uma grande cadeia de transmissão do bacilo na comunidade e as dificuldades dos programas de saúde na vigilância e controle da doença (PIRES CAA, 2012) além de sugerir que ainda existe grande número de doentes transmissores não diagnosticados, o que dificulta a eliminação da doença no estado (SOBRINHO RAS e MATHIAS TAF, 2008).

Assim como a taxa de detecção, a prevalência também vinha apresentando poucas variações até o último ano do período estudado, quando houve redução da classificação deste indicador de "alta" para "média" prevalência. Tal mudança está de acordo com a tendência observada pela OMS em 2015, em âmbito global (WHO, 2016).

Além disso, também corrobora a tendência observada pelo Ministério da Saúde no Brasil, nos últimos anos, na qual se registram decréscimos contínuos nas taxas de prevalência e de detecção de casos novos de hanseníase (BOECHAT N e PINHEIRO LCS, 2012).

No entanto, é importante ressaltar que, mesmo com a queda da taxa observada neste estudo, a prevalência continua acima do desejado pelo Ministério da Saúde que tinha o compromisso de eliminação da hanseníase como problema de saúde pública até o ano de 2015, com a meta de alcançar menos de um caso por 10.000 habitantes (OMS, 2010).

A proporção de casos diagnosticados com grau II de incapacidade física é utilizada como forma de avaliar a capacidade dos programas de saúde em reconhecer e tratar a doença em estágios iniciais, visto que a prevenção das incapacidades é um dos objetivos principais do programa de combate contra a hanseníase (RIBEIRO GC, et al, 2014).

Durante o período analisado por este estudo, os valores deste indicador sofreram poucas variações, apresentando média proporção, estando de acordo com os valores divulgados pela OMS em escala global nos últimos anos (WHO, 2016) e semelhante ao encontrado em outras regiões do Brasil, como demonstrado no estudo em 2011 (RIBEIRO GC, et al, 2014) realizado na microrregião de Araçuaí em Minas Gerais e em uma pesquisa de 2012 (LANA FCF, et al, 2011) em Dourado/MS.

Por meio da pesquisa em questão, os dados demonstram que há um atraso quanto ao diagnóstico da hanseníase. Assim, pode-se afirmar que há a sugestão sobre os serviços de saúde da região não estariam conseguindo captar todos os pacientes com Hanseníase existentes. Isso contribuiria para que ocorresse a permanência de casos não diagnosticados, isto é, continuidade da cadeia de transmissão e diagnósticos com grau de incapacidade (OLIVEIRA KS, et al, 2015).

Somado a isso, existe a proporção de curados no ano com incapacidades físicas, a qual é outro indicador de saúde, que pode demonstrar dados, a fim de programação de políticas voltadas ao controle de sequelas, bem como disponibilização de recursos para atender pacientes já com incapacidades físicas quando diagnosticados (BRASIL, 2010).

Apesar de ser um indicador importante (LIMA LS, et al, 2010; MONTEIRO LD, et al, 2013; FINEZ MA e SALOTTI SRA, 2001) no presente estudo após a alta da poliquimioterapia, essa proporção não pôde ser calculada, tendo em vista que segundo a orientação do Ministério da Saúde, tal indicador só pode ser mensurado se o percentual de avaliação de contatos na alta for igual ou superior a $75 \%$, e em nenhum momento do período estudado alcançou este patamar, indicando uma fragilidade do serviço de saúde.

Em 2013, houve identificação que vários estados do Nordeste, no período de 2001 a 2010, apresentaram valores classificados como regulares ou precários de cura entre os casos novos diagnosticados. Verificou-se neste estudo que no período entre 2010 e 2015 a proporção de cura foi regular (UNB, 2014).

Em um estudo de 2015 mostrou um índice de abandono classificado como bom em três cidades do sul do país (OLIVEIRA KS, et al, 2015)., assim como evidenciado no presente estudo. Enquanto no Sudeste estudos revelaram taxas de $15 \%$ de abandono (SOUSA MWG, et al, 2012). 
Porém, é importante considerar que o abandono de tratamento ainda é um dos empecilhos para o controle da hanseníase e estes valores estão subestimados nos dados epidemiológicos, podendo não refletir a realidade (ROMÃO ER e MAZZONI AM, 2013).

Os contatos intradomiciliares de pacientes de hanseníase têm risco aumentado de desenvolver a doença, portanto sua busca é indispensável (OMS, 2010). No país, o percentual de exame de contatos considerada regular em 2010, com 58\% (BRASIL, 2012). Este estudo apresentou dados que concordam com a taxa nacional, de precária e regular avaliação dos contatos intradomiciliares. Isso demonstra que há necessidade de maior fiscalização na realização das diversas etapas do controle da doença.

As cidades do estado do Pará em que se verificaram maiores taxas de incidência anual e total de Hanseníase, contabilizando todos os anos do estudo, foram Marituba, Belém, Marabá, Parauapebas e Altamira. Marituba e Belém fazem parte da mesorregião Metropolitana do estado do Pará, enquanto Parauapebas e Marabá constituem a mesorregião Sudeste do estado. Altamira faz parte da mesorregião sudoeste e suas altas taxas de incidência podem ser relacionadas ao desenvolvimento econômico e aumento populacional desordenado, causados pela construção da Hidrelétrica de Belo Monte, em 2009 (OLIVEIRA VM, et al, 2013).

A mesorregião Sudeste, registrou o maior número de casos no estado do Pará. Tais dados podem estar também relacionados ao desenvolvimento e expansão populacional desta região, pela construção das eclusas da Hidrelétrica de Tucuruí e a construção da Hidrelétrica de Marabá, além da presença da empresa de mineração Vale, responsável pela implantação da siderúrgica "Aços Laminados do Pará" (ALPA) em Marabá e pela proliferação de atividades mineradoras em municípios como Canaã dos Carajás, Curionópolis, Eldorado dos Carajás, além de Marabá e Parauapebas (BRASIL, 2010).

Já o considerável número de casos na região Metropolitana de Belém, pode ser atribuído à alta densidade demográfica e existência de melhores centros de referências de saúde na área, o que favorece a detecção de novos casos de hanseníase, assim como o manejo e manutenção de todos os pacientes registrados no SINAN (IGNOTTI E, et al, 2001). Ou seja, todas estas cidades representam centros de atração populacional e desenvolvimento urbano no estado do Pará.

A distribuição média de incidência sobre Hanseníase em função da faixa etária mostrou-se consideravelmente elevada em maiores de 15 anos. Este dado corrobora com a tendência mundial apresentada pela OMS nos últimos anos (WHO, 2016).

E também está de acordo com o demonstrado pela literatura, em território nacional (BRASIL, 1994; IBGE, 2014; IBGE, 2015; ROMÃO ER e MAZZONI AM, 2013).

A hanseníase é considerada uma doença de adultos pelo longo período de incubação pelo M. Leprae. Além disso, deve-se considerar que a maioria dos pacientes com idade acima de 15 anos, se encontra na fase economicamente ativa, tendo, portanto, maior exposição a um potencial contágio (IBGE, 2014).

Quanto à distribuição média de acordo com gênero, obteve-se predomínio da enfermidade no sexo masculino. Essa tendência também é descrita mundialmente pela OMS, que divulgou proporção semelhante à do presente estudo durante o mesmo período estudado (WHO, 2016).

Da mesma forma, este cenário também foi observada em outros cenários nas várias regiões do Brasil, como demonstrado por Monteiro LD, et al (2013), Sarmento APA, et al (2015) e Romão ER e Mazzoni AM (2013) sendo que o maior risco de exposição nesta população é amplamente reconhecido.

Para explicar esta realidade, alguns fatores podem ser considerados, tais como a menor procura aos serviços de saúde por parte da população masculina e uma menor preocupação dos homens em relação à saúde e a estética (SARMENTO APA, et al, 2015).

Fatores estes que se relacionam com uma demora no diagnóstico da hanseníase e geram acúmulo de casos para esse gênero. Contrariamente então, as mulheres teriam mais oportunidade de diagnóstico precoce (BRASIL, 1994). 
No entanto, é importante salientar que a preponderância do sexo masculino na hanseníase não é universal, já que existem várias áreas onde a ocorrência da hanseníase é igual nos dois sexos, ou ocasionalmente até mesmo maior entre as mulheres (ROMÃO ER e MAZZONI AM, 2013).

A classificação operacional da hanseníase revelou que a ocorrência de casos multibacilares no estado foi predominante. Esta proporção obtida é semelhante à média mundial demonstrada pela OMS (WHO, 2016) e também está de acordo com estudos nacionais como os realizados por Brito KKG, et al (2015), Miranzi SSC, et al (2010), Romão ER e Mazzoni AM (2013), Lima LS, et al (2010).

Este achado indica a presença de casos avançados de hanseníase, ou seja, das formas graves da doença, e refletem, indiretamente, a magnitude da infecção na comunidade, onde a presença de bacilos circulantes é intensa (WHO, 2016).

O que, por sua vez, é uma forte evidência de que a doença vem sendo diagnosticada tardiamente e que a cadeia de transmissão do $\mathrm{M}$. leprae continua a ocorrer, servindo de alerta pelo grande potencial infectante e incapacitante, característico destes casos multibacilares (ROMÃO ER e MAZZONI AM, 2013).

Também é válido considerar que o predomínio da classificação operacional multibacilar pode refletir a falta de preparo técnico no diagnóstico, a dificuldade de acesso precoce aos serviços de saúde e a possibilidade de que a estrutura atualmente mobilizada no combate à hanseníase esteja trabalhando de maneira estática, com uma demanda passiva de pacientes já antigos (LIMA LS, et al, 2010).

Concordando com este predomínio da classificação multibacilar, o presente estudo, também observou preponderância do esquema terapêutico poliquimioterápico multibacilar, com 12 doses, durante toda a série histórica analisada por Salles BO, et al (2015) em Campinas/SP e Pinto RA, et al (2010) em Salvador/BA.

Quanto à forma clínica, a distribuição mostrou predominância para a classificação Dimorfa, concordando com a classificação multibacilar predominante, visto que as formas indeterminadas e tuberculoide são normalmente associadas à classificação operacional paucibacilar. Enquanto as formas dimorfa e virchowiana, são mais associadas à classificação multibacilar (LIMA LS, et al, 2010).

Este predomínio é condizente com diversos estudos nacionais, como os realizados por Lima LS, et al (2010), Romão ER e Mazzoni AM (2013), Lima LS, et al (2009) Monteiro LD, et al (2013) e Lana FCF, et al (2011), corroborando com a hipótese de diagnóstico tardio e manutenção na cadeia de disseminação da doença, permitindo inferir novamente, que a rede básica de saúde não vem detectando os casos nas formas precoces.

O que, por sua vez, torna mais provável o desenvolvimento de incapacidades físicas, lesões, estado reacional, e provoca um possível afastamento da população economicamente ativa de suas atividades, gerando grande custo social (MONTEIRO LD, et al, 2013).

Quanto aos tipos de saída, pode-se observar que a cura foi predominante em todos os anos, como esperado.

Porém, é importante destacar que houve um número considerável de campos não preenchidos, inclusive superando as outras formas de saída, com exceção da cura.

Isso demonstra uma falha por parte das equipes responsáveis pelo registro dos dados, indicando que há necessidade de maior esclarecimento destes profissionais quanto à importância do preenchimento de todas as informações durante o seguimento dos pacientes, visto que esses dados são fundamentais para o planejamento e aprimoramento das ações de controle da Hanseníase.

No estado do Pará as regiões com maior número de notificações foram a Região Metropolitana de Belém e o Sudeste do estado, representados pelos municípios de Marituba, Belém, Marabá e Parauapebas.

Além destes, o município de Altamira, que faz parte da região Sudoeste, também se destaca com um alto número de casos novos. Juntos, estes municípios totalizam cerca de $30 \%$ de todos os casos novos registrados no período estudado. 


\section{CONCLUSÃO}

Conclui-se que no estado do Pará as regiões com maior número de notificações foram a Região Metropolitana de Belém e o Sudeste do estado, representados pelos municípios de Marituba, Belém, Marabá e Parauapebas. Além destes, o município de Altamira, que faz parte da região Sudoeste, também se destaca com um alto número de casos novos. Juntos, estes municípios totalizam cerca de $30 \%$ de todos os casos novos registrados no período estudado. O perfil clínico-epidemiológico recente dos casos novos de Hanseníase constitui-se predominantemente de homens $(60 \%)$, de classificação operacional multibacilar (64\%), forma clínica dimorfa (45\%) e maiores de 15 anos (89\%), embora a porcentagem de casos em menores de 15 anos seja significativa (11\%). No que tange à classificação operacional, a proporção de casos novos diagnosticados com o grau de incapacidade física avaliado foi qualificada como bom em todos os anos. A proporção de cura entre casos novos diagnosticados foi precária. A proporção de casos curados com grau de incapacidade física avaliado também foi precária, contudo, apenas no ano de 2012 apresentou classificação regular. A proporção de examinados entre os contatos intradomiciliares de casos novos diagnosticados foi regular na maioria dos anos, com exceção de 2013, que foi considerado bom. E, por fim, a proporção de abandono do tratamento na prevalência foi avaliada como bom em toda a série histórica estudada.

\section{AGRADECIMENTOS E FINANCIAMENTO}

Agradecimento à equipe da Secretaria de Estado de Saúde Pública do Pará pela colaboração em ceder os dados para a pesquisa.

\section{REFERÊNCIAS}

1. ALVES ED, et al. Hanseníase: avanços e desafios. Brasília, 2014.

2. BOECHAT N, PINHEIRO LCS. A Hanseníase e a sua Quimioterapia. Rev. Virtual Quim., v. 4, n. 3, p. $247-256.2012$.

3. BRASIL. Guia de Vigilância em Saúde. Secretaria de Vigilância em Saúde. Brasília: Ministério da Saúde, 2014. p. 812.

4. BRASIL. Ministério da Saúde. Secretaria de Vigilância em Saúde. Departamento de Vigilância das Doenças Transmissíveis. Exercício de Monitoramento da Eliminação da hanseníase no Brasil. Brasília: MS, 2015.

5. BRASIL. Secretaria de Estado de Saúde do Distrito Federal. Protocolo de Atendimento - Brasília: Subsecretaria de Vigilância à Saúde. Brasília: MS, 2007.

6. BRASIL. Ministério da Saúde, Secretaria de Atenção à Saúde. Departamento de Atenção Básica. Série A. Normas e Manuais Técnicos. Cadernos de Atenção Básica. Brasília: Ministério da Saúde, 2008.

7. BRASIL. Ministério da Saúde. Secretaria de Vigilância em Saúde. Departamento de Vigilância Epidemiológica. Guia de procedimentos técnicos: baciloscopia em hanseníase. Brasília: MS, 2010.

8. BRASIL. Ministério da Saúde. Secretaria de Vigilância em Saúde. Departamento de Vigilância das Doenças Transmissíveis. Plano Integrado de Ações Estratégicas. Brasília, 2012.

9. BRASIL. Ministério da Saúde. Secretaria Nacional de Programas Especiais. Divisão Nacional de Dermatologia Sanitária. Relatório do grupo tarefa sobre aspectos sociais. Brasilia: MS, 1994.

10. BRITO KKG, et al. Análise epidemiológica da hanseníase em um estado endêmico do nordeste brasileiro. Rev Gaúcha Enferm., v. 36, p. 24-30. 2015.

11. FINEZ MA, SALOTTI SRA. Identificação do grau de incapacidades em pacientes portadores de hanseníase através da avaliação neurológica simplificada. J Health Sci Inst., v. 29, n. 3, p. 171-5. 2001.

12. IBGE - Instituto Brasileiro de Geografia e Estatística. Produto Interno Bruto dos Municípios 2010 - 2014.

13. IBGE - Instituto Brasileiro de Geografia e Estatística. Estimativas populacionais para os municípios e para as Unidades da Federação brasileiros em 2015.

14. IGNOTTI E, et al. Estudo da adesão ao tratamento da hanseníase no município de Duque de Caxias - Rio de Janeiro. Hansen. Int., v.26, n. 1, p. 23-30. 2001.

15. LANA FCF, et al. Perfil epidemiológico da hanseníase na microrregião de Araçuaí e sua relação com ações de controle. Esc Anna Nery, v.15, n. 1, p. 62-67, jan-mar. 2011.

16. LASTÓRIA JC, ABREU MAMM. Hanseníase: diagnóstico e tratamento. Diagn Tratamento. v. 17, n. 4, p. $173-9.2012$. 
17. LIMA LS, et al. Caracterização clínica-epidemiológica dos pacientes diagnosticados com Hanseníase no município de Caxias, MA. Ver Bras Clin Med., v. 7, p. 74-83. 2009.

18. LIMA LS, et al. Perfil epidemiológico dos pacientes com hanseníase atendidos em Centro de Saúde em São Luís, MA. Ver Bras Clin Med., v. 8, n. 4, p. 323-7. 2010.

19. MIRANZI SSC, et al. Perfil epidemiológico da hanseníase em um município brasileiro, no período de 2000 a 2006. Ver Soc Bras Med Trop., v. 43, n. 1, p. 62-67, jan-fev. 2010.

20. MONTEIRO LD, et al. Incapacidades físicas em pessoas acometidas pela hanseníase no período pós-alta da poliquimioterapia em um município no Norte do Brasil. Cad. Saúde Pública, Rio de Janeiro, v. 29, n. 5, p. 909-920, mai, 2013.

21. OLIVEIRA KS, et al. Avaliação dos indicadores epidemiológicos e operacionais para a hanseníase em municípios prioritários no estado do Paraná, 2001 a 2010. Epidemiol. Serv. Saúde, Brasília, v. 24, n. 3, p. 507-516, jul-set. 2015.

22. OLIVEIRA VM, et al. Levantamento epidemiológico da hanseníase no nordeste brasileiro durante o período de 2001 2010. Scire Salutis, v. 3, n.1, Out, Nov, dez 2012, Jan, Fev, Mar 2013.

23. ORGANIZAÇÃO MUNDIAL DE SAÚDE. Estratégia Global Aprimorada para Redução Adicional da Carga da Hanseníase. 2010.

24. PALÁCIOS VRCM, et al. Estudo da situação da hanseníase no estado do Pará. Revista Paraense de Medicina, v. 24, n. 2, abril-junho. 2010.

25. PINTO RA, et al. Perfil clínico e epidemiológico dos pacientes notificados com hanseníase em um hospital especializado em Salvador, Bahia. Ver B.S. Publica Miolo., v. 34, n. 4, p. 906- 918. 2011.

26. PIRES CAA, et al. Hanseníase em menores de 15 anos: a importância do exame de contato. Rev Paul Pediatr., v. 30, n. 2, p. 292-5. 2012.

27. POLIT DF, et al. Fundamentos de Pesquisa em Enfermagem: métodos, avaliação e utilização. 5 ed. Porto Alegre: Artmed, 2004.

28. RIBEIRO GC, et al. Estimativa da prevalência oculta da hanseníase na microrregião de Diamantina - Minas Gerais. Rev. Eletr. Enf., v. 16, n. 4, p. 728-35, out/dez. 2014.

29. RIDLEY DS, JOPLING WH. Classification of Leprosy According to Immunity. A Five-group System International Journal of Leprosy., v. 34, n. 3. p. 255-273. 1966.

30. RODUIGUES LC, LOCKWOOD DN. Leprosy now: epidemiology, progress, challenges, and research gaps. Lancet Infect Dis., v. 11, n. 6, p. 464-70, jun.2011.

31. ROMÃO ER, MAZZONI AM. Perfil epidemiológico da hanseníase no município de Guarulhos, SP. Ver Epidemiol Control Infect., v. 3, n. 1, p. 22-27. 2013.

32. SALLES BO, et al. Perfil epidemiológico da hanseníase em Hospital Universitário de Campinas, SP: explorando fichas de notificação. Hansen Int., v. 40, n. 2, p. 36-47. 2015.

33. SARMENTO APA, et al. Perfil epidemiológico da hanseníase no período de 2009 a 2013 no município de Montes Claros (MG). Ver Soc Bras Clin Med., v. 13, n. 3, p.180-4, jul-set. 2015.

34. SOBRINHO RAS, MATHIAS TAF. Perspectivas de eliminação da hanseníase como problema de saúde pública no Estado do Paraná, Brasil. Cad. Saúde Pública, Rio de Janeiro, v. 24, n. 2, p. 303-314, fev. 2008.

35. SOUSA MWG, et al. Perfil epidemiológico da hanseníase no estado do Piauí, período de 2003 a 2008 . Na Bras Dermatol., v. 87, n. 3, p. 401-7. 2012.

36. UNIVERSIDADE DE BRASÍLIA. Núcleo de Estudos em Educação e Promoção da Saúde. Hanseníase. Avanços e desafios. Brasília, DF, 2014. 492 p.

37. WHO. Global Strategy for Further Reducing the Leprosy Burden and Sustaining Leprosy Control Activities. Regional Office for South-EastAsia New Delhi.2006.

38. WHO. Weekly epidemiological record World Health Organization. Global leprosy update, 2015: time for action, account ability and inclusion. 2016. 